\title{
Cefcapene Pivoxil
}

National Cancer Institute

\section{Source}

National Cancer Institute. Cefcapene Pivoxil. NCI Thesaurus. Code C98218.

The pivalate ester prodrug form of cefcapene, a semi-synthetic third-generation cephalosporin with antibacterial activity. After oral administration of cefcapene pivoxil, the ester bond is cleaved, releasing active cefcapene. 